TRANSACTIONS OF THE

AMERICAN MATHEMATICAL SOCIETY

Volume 355, Number 5 , Pages 1843-1856

S 0002-9947(02)03125-2

Article electronically published on December 2, 2002

\title{
THE STRINGY E-FUNCTION OF THE MODULI SPACE OF RANK 2 BUNDLES OVER A RIEMANN SURFACE OF GENUS 3
}

\author{
YOUNG-HOON KIEM
}

\begin{abstract}
We compute the stringy E-function (or the motivic integral) of the moduli space of rank 2 bundles over a Riemann surface of genus 3 . In doing so, we answer a question of Batyrev about the stringy E-functions of the GIT quotients of linear representations.
\end{abstract}

\section{Statement of the main Result}

The stringy E-function is an invariant for singular varieties, due to Kontsevich, Batyrev, Denef and Loeser, which retains useful information about the singularities (see [Bat, DL1, DL2], [Cra, Loo]).

Let $X$ be a variety with at worst log-terminal singularities, i.e.,

- $X$ is $\mathbb{Q}$-Gorenstein, and

- for a resolution of singularities $\rho: Y \rightarrow X$ such that the exceptional locus of $\rho$ is a divisor $D$ whose irreducible components $D_{1}, \cdots, D_{r}$ are smooth divisors with only normal crossings, we have

$$
K_{Y}=\rho^{*} K_{X}+\sum_{i=1}^{r} a_{i} D_{i}
$$

with $a_{i}>-1$ for all $i$, where $D_{i}$ runs over all irreducible components of $D$. The divisor $K_{Y}-\rho^{*} K_{X}$ is called the discrepancy divisor.

For each subset $J \subset I=\{1,2, \cdots, r\}$, define $D_{J}=\bigcap_{j \in J} D_{j}, D_{\emptyset}=Y$ and $D_{J}^{0}=D_{J} \backslash \bigcup_{j \in I \backslash J} D_{j}$. Then the stringy E-function of $X$ is defined by

$$
E_{s t}(X ; u, v)=\sum_{J \subset I} E\left(D_{J}^{0} ; u, v\right) \prod_{j \in J} \frac{u v-1}{(u v)^{a_{j}+1}-1},
$$

where

$$
E(Z)=\sum_{p, q} \sum_{k \geq 0}(-1)^{k} h^{p, q}\left(H_{c}^{k}(Z ; \mathbb{C})\right) u^{p} v^{q}
$$

is the Hodge-Deligne polynomial for a variety $Z$.

The "change of variable formula" (Theorem 6.27 in Bat, Lemma 3.3 in [DL1]) implies that the function $E_{s t}$ is independent of the choice of a resolution. In particular, if $\rho$ is a crepant resolution (i.e., $\left.\rho^{*} K_{X}=K_{Y}\right)$, then $E_{s t}(X ; u, v)=E(Y ; u, v)$.

Received by the editors November 1, 2001.

2000 Mathematics Subject Classification. Primary 14F05, 14F43, 14J10.

(C)2002 American Mathematical Society 
A projective $\mathbb{Q}$-Gorenstein algebraic variety of dimension $d$ with at worst logterminal singularities has the Poincaré duality

$$
E_{s t}(X ; u, v)=(u v)^{d} E_{s t}\left(X ; u^{-1}, v^{-1}\right)
$$

with $E_{s t}(X ; 0,0)=1$ (Theorem 3.7 in [Bat]).

In this paper, we compute the stringy E-function of the moduli space $\mathcal{N}$ of rank 2 bundles of even degree over a Riemann surface of genus 3 with fixed determinant 1 Our main result is the following:

Theorem 1.1.

$$
\begin{aligned}
E_{s t}(\mathcal{N})= & \frac{\left(1-u^{2} v\right)^{3}\left(1-u v^{2}\right)^{3}-(u v)^{4}(1-u)^{3}(1-v)^{3}}{(1-u v)\left(1-(u v)^{2}\right)} \\
& -\frac{(u v)^{2}}{2}\left(\frac{(1-u)^{3}(1-v)^{3}}{1-u v}-\frac{(1+u)^{3}(1+v)^{3}}{1+u v}\right) \\
& +2^{6}(u v)^{5}\left(1+u v+(u v)^{2}\right)\left(1+(u v)^{2}\right)\left(\frac{u v-1}{(u v)^{5}-1}\right)^{2} .
\end{aligned}
$$

The stringy Euler number is

$$
e_{s t}(\mathcal{N})=\lim _{u, v \rightarrow 1} E_{s t}(\mathcal{N})=31 \frac{9}{25} .
$$

The deepest singularities in the moduli space are the geometric invariant theory (GIT) quotient $s l(2)^{3} / / S L(2)$, where the action is the diagonal adjoint action. This is a hypersurface singularity, and that makes the genus 3 case special. Batyrev asked (Question 5.5 in [Bat]) the following:

Question (Batyrev): Let $X$ be a GIT quotient of $\mathbb{C}^{n}$ modulo a linear action of $G \subset S L(n)$. Is it true that $E_{s t}(X ; u, v)$ is a polynomial?

He showed that this is true when $G$ is abelian or finite. A corollary of our computation is that the answer is NO in general.

\section{Corollary 1.2.}

$$
\begin{aligned}
E_{s t}\left(\mathbb{C}^{9} / / S L(2)\right) & =E\left(\left[\mathbb{C}^{9} / / S L(2)\right]^{s}\right)+\frac{(u v)^{3}\left(1+u v+(u v)^{2}\right)}{1+u v} \\
& +(u v)^{5}\left(1+u v+(u v)^{2}\right)\left(1+(u v)^{2}\right)\left(\frac{u v-1}{(u v)^{5}-1}\right)^{2},
\end{aligned}
$$

where $\left[\mathbb{C}^{9} / / S L(2)\right]^{s}$ denotes the smooth part of $\mathbb{C}^{9} / / S L(2)$.

Since $E\left(\left[\mathbb{C}^{9} / / S L(2)\right]^{s}\right)$ is a polynomial, we deduce that the stringy E-function of $\mathbb{C}^{9} / / S L(2)$ is not a polynomial.

When the genus of the Riemann surface is 2, the moduli space is isomorphic to $\mathbb{P}^{3}$ and thus the E-function is $1+u v+(u v)^{2}+(u v)^{3}$. When the genus is greater than 3 , the deepest singularities are no longer hypersurface singularities and it doesn't seem possible to find the discrepancy divisor by explicit computation as in this paper.

In $\S 2$, we study the singularities of the moduli space $\mathcal{N}$. In $\S \S 3,4,5$, we work out the blow-ups to get a desingularization of $\mathcal{N}$. We compute the discrepancy divisor in $\S 6$, and we prove Theorem 1.1 and Corollary 1.2 in $\S 7$. We conclude this paper with a formula for the stringy E-function of the moduli space $\mathcal{M}$ of rank 2 bundles of even degree, without fixing determinant, over a Riemann surface of genus 3.

\footnotetext{
${ }^{1}$ See [New], Ses] for general facts about the moduli space.
} 


\section{The Moduli space}

The moduli space $\mathcal{N}$ of rank 2 semistable bundles of degree 0 with trivial determinant over a Riemann surface of genus $g=3$ is a singular projective variety of complex dimension 6. The singularities are Gorenstein by Theorem A of [DN] and log-terminal as we will see in $\S 6$. We refer to [New], Ses, Ki1 for general results on the moduli space.

The singular locus in $\mathcal{N}$ is the Kummer variety $\mathcal{K}$, which corresponds to those rank 2 bundles $L \oplus L^{-1}$ for some line bundle $L$ of degree 0 . The involution $L \rightarrow L^{-1}$ gives us a $\mathbb{Z}_{2}$ action on the Jacobian $J a c_{0}$, and the Kummer variety $\mathcal{K}$ is identified with $J a c_{0} / \mathbb{Z}_{2}$. There are $2^{2 g}$ fixed points $\mathbb{Z}_{2}^{2 g}=\left\{\left[L \oplus L^{-1}\right]: L \cong L^{-1}\right\}$. Thus we have a stratification

$$
\mathcal{N}=\mathcal{N}^{s} \sqcup\left(\mathcal{K}-\mathbb{Z}_{2}^{2 g}\right) \sqcup \mathbb{Z}_{2}^{2 g} .
$$

The moduli space $\mathcal{N}$ is constructed as the GIT quotient of a smooth quasiprojective variety $\mathfrak{R}$, which is a subset of the space of holomorphic maps from the Riemann surface to the Grassmannian $G r(2, p)$ of 2-dimensional quotients of $\mathbb{C}^{p}$, where $p$ is a large even number, by the action of $G=S L(p)$. By deformation theory, the slice at a point $h \in \mathfrak{R}$, which represents $L \oplus L^{-1}$ where $L \cong L^{-1}$, is

$$
H^{1}\left(\operatorname{End}_{0}\left(L \oplus L^{-1}\right)\right) \cong H^{1}(\mathcal{O}) \otimes s l(2)
$$

where the subscript 0 denotes the trace-free part. According to Luna's slice theorem, there is a neighborhood of the point $\left[L \oplus L^{-1}\right]$ with $L \cong L^{-1}$, isomorphic to $H^{1}(\mathcal{O}) \otimes s l(2) / / S L(2)$ since the stabilizer of the point is $S L(2)$ (Ki1] (3.3)). Because $\operatorname{dim} H^{1}(\mathcal{O})=g$, the deepest singularities are just

$$
s l(2)^{g} / / S L(2)=\operatorname{Spec} \mathbb{C}\left[z_{1}, \cdots, z_{3 g}\right]^{S L(2)} .
$$

By the classical invariant theory (see Wey], or more precisely [Hue, 5.1), there is an explicit description of the generators and relations of the invariant subring $\mathbb{C}\left[z_{1}, \cdots, z_{3 g}\right]^{S L(2)}$. The special feature of the case $g=3$ is that the quotient $X:=\operatorname{sl}(2)^{g} / / S L(2)$ is a hypersurface: For each $\left(\vec{u}_{1}, \vec{u}_{2}, \vec{u}_{3}\right) \in \operatorname{sl}(2)^{3}$, let $x_{1}=\vec{u}_{1} \cdot \vec{u}_{1}, x_{2}=\vec{u}_{2} \cdot \vec{u}_{2}, x_{3}=\vec{u}_{3} \cdot \vec{u}_{3}, x_{4}=\vec{u}_{1} \cdot \vec{u}_{2}, x_{5}=\vec{u}_{1} \cdot \vec{u}_{3}, x_{6}=$ $\vec{u}_{2} \cdot \vec{u}_{3}, x_{7}=\operatorname{det}\left(\vec{u}_{1}, \vec{u}_{2}, \vec{u}_{3}\right)$. Then $\mathbb{C}^{9} / / S L(2)$ is the hypersurface of $\mathbb{C}^{7}=$ Spec $\mathbb{C}\left[x_{1}, x_{2}, \cdots, x_{6}, x_{7}\right]$ given by the equation

$$
f\left(x_{1}, \cdots, x_{7}\right)=x_{1} x_{2} x_{3}+2 x_{4} x_{5} x_{6}-x_{1} x_{6}^{2}-x_{2} x_{5}^{2}-x_{3} x_{4}^{2}-x_{7}^{2} .
$$

The locus of $\mathcal{K}$ in this neighborhood, as a set, is given by

$$
x_{7}=0, \quad x_{1} x_{2}-x_{4}^{2}=0, \quad x_{1} x_{3}-x_{5}^{2}=0, \quad x_{1} x_{6}-x_{4} x_{5}=0,
$$

because a point in $\mathcal{K}_{X}:=\mathcal{K} \cap X$ can be represented by the $\mathbb{C}^{*}$-fixed points $\left(\vec{u}_{1}, \vec{u}_{2}, \vec{u}_{3}\right)$ with $\vec{u}_{i} \in\{\operatorname{diag}(a,-a): a \in \mathbb{C}\}, i=1,2,3$.

Next, we consider the middle stratum $\mathcal{K}-\mathbb{Z}_{2}^{2 g}$. Once again, if we consider a point $h \in \mathfrak{R}$ representing $L \oplus L^{-1}$ with $L \nsubseteq L^{-1}$, the slice to the orbit is isomorphic to

$$
H^{1}\left(\operatorname{End}_{0}\left(L \oplus L^{-1}\right)\right) \cong H^{1}(\mathcal{O}) \oplus H^{1}\left(L^{2}\right) \oplus H^{1}\left(L^{-2}\right)
$$

The stabilizer $\mathbb{C}^{*}$ acts with weights $0,2,-2$ respectively on the components. Hence, there is a neighborhood of the point $\left[L \oplus L^{-1}\right] \in \mathcal{K}-\mathbb{Z}_{2}^{2 g}$ in $\mathcal{N}$, isomorphic to

$$
H^{1}(\mathcal{O}) \oplus\left(H^{1}\left(L^{2}\right) \oplus H^{1}\left(L^{-2}\right) / / \mathbb{C}^{*}\right) .
$$

Notice that $H^{1}(\mathcal{O})$ is the tangent space to $\mathcal{K}$, and hence

$$
H^{1}\left(L^{2}\right) \oplus H^{1}\left(L^{-2}\right) / / \mathbb{C}^{*}=\mathbb{C}^{2 g-2} / / \mathbb{C}^{*}
$$


is the normal cone. The GIT quotient of the projectivization $\mathbb{P C}^{2 g-2}$ by the induced $\mathbb{C}^{*}$ action is $\mathbb{P}^{g-2} \times \mathbb{P}^{g-2}$, and the normal cone $\mathbb{C}^{2 g-2} / / \mathbb{C}^{*}$ is obtained by collapsing the zero section of the line bundle $\mathcal{O}_{\mathbb{P}^{g-2} \times \mathbb{P}^{g-2}}(-1,-1)$.

\section{FiRST BLOW-UP}

We will desingularize the moduli space $\mathcal{N}$ by blowing up three times. In this section, we describe the first blow-up.

Let $\mathcal{N}_{1}$ be the blow-up of $\mathcal{N}$ along the deepest strata $\mathbb{Z}_{2}^{2 g}$, and let $D_{1}^{\prime}$ be the exceptional divisor. Since the deepest singularities are all $X:=\mathbb{C}^{9} / / S L(2)$, we consider only one of them. The GIT quotient $X$ is the hypersurface of $\mathbb{C}^{7}$ with the equation

$$
f\left(x_{1}, \cdots, x_{7}\right)=x_{1} x_{2} x_{3}+2 x_{4} x_{5} x_{6}-x_{1} x_{6}^{2}-x_{2} x_{5}^{2}-x_{3} x_{4}^{2}-x_{7}^{2} .
$$

We blow up at the origin and denote the exceptional divisor also by $D_{1}^{\prime}$. In terms of a local chart, the blow-up map is

$$
\left(y_{1}, \cdots, y_{7}\right) \rightarrow\left(y_{1}, y_{1} y_{2}, \cdots, y_{1} y_{7}\right) .
$$

We have $f\left(x_{1}, \cdots, x_{7}\right)=y_{1}^{2} g_{1}\left(y_{1}, \cdots, y_{7}\right)$, where

$$
g_{1}\left(y_{1}, \cdots, y_{7}\right)=y_{1}\left(y_{2} y_{3}+2 y_{4} y_{5} y_{6}-y_{6}^{2}-y_{2} y_{5}^{2}-y_{3} y_{4}^{2}\right)-y_{7}^{2} .
$$

Hence, the blow-up $X_{1}$ is the hypersurface given by $g_{1}$, and the exceptional divisor $D_{1}^{\prime}$ is the subset $y_{1}=0, y_{7}=0$ in the local chart. Let $\tilde{\mathcal{K}}_{X}$ be the proper transform of $\mathcal{K}_{X}$.

The singular set of $X_{1}$ in this chart is, by solving $\nabla g_{1}=0$, the union of

$$
y_{1}=0, \quad y_{7}=0, \quad y_{2} y_{3}+2 y_{4} y_{5} y_{6}-y_{6}^{2}-y_{2} y_{5}^{2}-y_{3} y_{4}^{2}=0
$$

and

$$
y_{7}=0, \quad y_{2}-y_{4}^{2}=0, \quad y_{3}-y_{5}^{2}=0, \quad y_{6}-y_{4} y_{5}=0 .
$$

Notice that the second component of the singular set is just the proper transform $\tilde{\mathcal{K}}_{X}$ in view of (3).

Now we switch to other charts. Since $x_{1}, x_{2}, x_{3}$ are symmetric, we consider, for instance,

$$
\left(y_{1}, \cdots, y_{7}\right) \rightarrow\left(y_{5} y_{1}, y_{5} y_{2}, y_{5} y_{3}, y_{5} y_{4}, y_{5}, y_{5} y_{6}, y_{5} y_{7}\right) .
$$

In this chart, $X_{1}$ is given by the equation

$$
g_{5}\left(y_{1}, \cdots, y_{7}\right)=y_{5}\left(y_{1} y_{2} y_{3}+2 y_{4} y_{6}-y_{1} y_{6}^{2}-y_{2}-y_{3} y_{4}^{2}\right)-y_{7}^{2} .
$$

and $D_{1}^{\prime}$ by $y_{5}=0, y_{7}=0$. The singular locus in this chart is the union of

$$
y_{5}=0, \quad y_{7}=0, \quad y_{1} y_{2} y_{3}+2 y_{4} y_{6}-y_{1} y_{6}^{2}-y_{2}-y_{3} y_{4}^{2}=0
$$

and

$$
y_{7}=0, \quad y_{1} y_{2}-y_{4}^{2}=0, \quad y_{1} y_{3}-1=0, \quad y_{1} y_{6}-y_{4}=0 .
$$

Again the second component is $\tilde{\mathcal{K}}_{X}$ by comparing with (3) 2

From the local descriptions (77), (11), we see that the first component of the singular set is the subvariety

$$
\Delta_{X}=\left\{\left(y_{1}: \cdots: y_{7}\right) \mid y_{7}=0, y_{1} y_{2} y_{3}+2 y_{4} y_{5} y_{6}-y_{1} y_{6}^{2}-y_{2} y_{5}^{2}-y_{3} y_{4}^{2}=0\right\}
$$

\footnotetext{
${ }^{2}$ There is one more chart $\left(y_{1}, \cdots, y_{7}\right) \rightarrow\left(y_{1} y_{7}, \cdots, y_{6} y_{7}, y_{7}\right)$, but it doesn't intersect with the exceptional divisor.
} 
of the projective space $\mathbb{P}^{6}$.

Lemma 3.1. $\Delta_{X} \cong \mathbb{P}^{2} \times \mathbb{P}^{2} / \mathbb{Z}_{2}$.

Proof. Define a morphism $\mathbb{P}^{2} \times \mathbb{P}^{2} \rightarrow \mathbb{P}^{6}$ by

$$
((x: y: z),(p: q: r)) \rightarrow(2 x p: 2 y q: 2 z r: x q+y p: x r+z p: y r+z q: 0) .
$$

In fact, this came from the identity

$$
\begin{aligned}
& (x t+y s+z u)(p t+q s+r u) \\
& \quad=2 x p \frac{t^{2}}{2}+2 y q \frac{s^{2}}{2}+2 z r \frac{u^{2}}{2}+(x q+y p) t s+(x r+z p) t u+(y r+z q) s u .
\end{aligned}
$$

Since $\mathbb{C}[t, s, u]$ is a UFD, the morphism is a 2:1 map whose image is precisely $\Delta_{X}$, as one can easily check.

The singular locus of $X_{1}$ is thus $\Delta_{X} \cup \tilde{\mathcal{K}}_{X}$. By direct computation, the singular locus $\mathbb{P}^{2}$ of $\Delta_{X}$ is the intersection $\Delta_{X} \cap \tilde{\mathcal{K}}_{X}$, which is the exceptional divisor of the proper transform $\tilde{\mathcal{K}}_{X} \rightarrow \mathcal{K}_{X}$. For instance, in terms of the local chart of (5), the singular locus of (7) is given by the equations of (7) and (8).

We denote by $\Delta$ the disjoint union of $2^{6} \Delta_{X}$ 's in the exceptional divisor in $\mathcal{N}_{1}$ having $2^{6}$ components. Then $\mathcal{N}_{1}$ is smooth away from $\Delta \cup \tilde{\mathcal{K}}$.

\section{SECOND BLOW-UP}

In this section, we consider the second blow-up. Namely, we blow up $\mathcal{N}_{1}$ along the proper transform $\tilde{\mathcal{K}}$ of $\mathcal{K}$. This is particularly important because it is the partial desingularization of $\mathcal{N}$, defined in Ki3.

Let $\mathcal{N}_{2}$ be the blow-up of $\mathcal{N}_{1}$ along $\tilde{\mathcal{K}}$. Let $D_{2}^{\prime}$ be the exceptional divisor and $\tilde{D}_{1}^{\prime}$ the proper transform of $D_{1}^{\prime}$ having $2^{2 g}$ connected components. We will describe $\mathcal{N}_{2}$ as the partial desingularization of $\mathcal{N}$. For more details on partial desingularization, we refer to Ki1] and [Ki3].

Let $H$ be a reductive subgroup of $G=S L(p)$ and define $Z_{H}^{s s}$ as the set of semistable points in $\mathfrak{R}$ fixed by $H$. Let $\mathfrak{R}_{1}$ be the blow-up of $\mathfrak{R}^{s s}$ along the smooth subvariety $G Z_{S L(2)}^{s s}$. Then by Lemma 3.11 in [Ki3], the GIT quotient $\mathfrak{R}_{1}^{s s} / / G$ is the first blow-up $\mathcal{N}_{1}$. The $\mathbb{C}^{*}$-fixed point set in $\Re_{1}^{s s}$ is the proper transform $\tilde{Z}_{\mathbb{C}^{*}}^{s s}$ of $Z_{\mathbb{C}^{*}}^{s s}$ and the quotient of $G \tilde{Z}_{\mathbb{C}^{*}}^{s s}$ by $G$ is $\tilde{\mathcal{K}}$. If we denote by $\mathfrak{R}_{2}$ the blow-up of $\mathfrak{R}_{1}^{s s}$ along the smooth subvariety $G \tilde{Z}_{\mathbb{C}^{*}}^{s s}=G \times_{N^{\mathbb{C}^{*}}} \tilde{Z}_{\mathbb{C}^{*}}^{s s}$, where $N^{\mathbb{C}^{*}}$ is the normalizer of $\mathbb{C}^{*}$, then the GIT quotient $\mathfrak{R}_{2}^{s s} / / G$ is our second blow-up $\mathcal{N}_{2}$, again by Lemma 3.11 of Ki3]. This is Kirwan's partial desingularization of $\mathcal{N}$ (see $\S 3$ in [Ki1]), which is an orbifold.

By applying the algorithm for Betti numbers described in Ki3, the Poincaré series $P\left(\mathcal{N}_{2}\right)=\sum_{k>0} t^{k} \operatorname{dim} H^{k}\left(\mathcal{N}_{2}\right)$ can be computed as follows. By [Ki2], the equivariant Poincaré series $P^{G}\left(\mathfrak{R}^{s s}\right)=\sum_{k \geq 0} t^{k} \operatorname{dim} H_{G}^{k}\left(\mathfrak{R}^{s s}\right)$ is given by the gaugetheoretic computation of Atiyah and Bott in $\S 11$ of $\mathrm{AB}$, and we get

$$
P^{G}\left(\Re^{s s}\right)=\frac{\left(1+t^{3}\right)^{6}-t^{8}(1+t)^{6}}{\left(1-t^{2}\right)\left(1-t^{4}\right)} .
$$

In order to get $\mathfrak{R}_{1}^{s s}$ we blow up $\mathfrak{R}^{s s}$ along $G Z_{S L(2)}^{s s}$ and delete the unstable strata. So we get

$$
P^{G}\left(\mathfrak{R}_{1}^{s s}\right)=P^{G}\left(\mathfrak{R}^{s s}\right)+2^{6}\left(\frac{t^{2}+t^{4}+\cdots+t^{16}}{1-t^{4}}-\frac{t^{10}\left(1+t^{2}+t^{4}\right)}{1-t^{2}}\right) .
$$


Now $\mathfrak{R}_{2}^{s s}$ is obtained by blowing up $\mathfrak{R}_{1}^{s s}$ along $G \tilde{Z}_{\mathbb{C}^{*}}^{s s}$ and deleting the unstable strata. Thus we have

$$
\begin{aligned}
P^{G}\left(\Re_{2}^{s s}\right)=P^{G}\left(\Re_{1}^{s s}\right) & +\left(t^{2}+t^{4}+t^{6}\right)\left(\frac{1}{2} \frac{(1+t)^{6}}{1-t^{2}}+\frac{1}{2} \frac{(1-t)^{6}}{1+t^{2}}+2^{6} \frac{t^{2}+t^{4}}{1-t^{4}}\right) \\
& -\frac{t^{4}\left(1+t^{2}\right)}{1-t^{2}}\left((1+t)^{6}+2^{6}\left(t^{2}+t^{4}\right)\right) .
\end{aligned}
$$

Because the stabilizers of the $G$ action on $\mathfrak{R}_{2}^{s s}$ are all finite, we have

$$
H_{G}^{*}\left(\mathfrak{R}_{2}^{s s}\right) \cong H^{*}\left(\mathfrak{R}_{2}^{s s} / G\right)=H^{*}\left(\mathcal{N}_{2}\right),
$$

and hence we deduce that

$$
\begin{aligned}
P\left(\mathcal{N}_{2}\right) & =\frac{\left(1+t^{3}\right)^{6}-t^{8}(1+t)^{6}}{\left(1-t^{2}\right)\left(1-t^{4}\right)} \\
& +2^{6}\left(\frac{t^{2}+t^{4}+\cdots+t^{16}}{1-t^{4}}-\frac{t^{10}\left(1+t^{2}+t^{4}\right)}{1-t^{2}}\right) \\
& +\left(t^{2}+t^{4}+t^{6}\right)\left(\frac{1}{2} \frac{(1+t)^{6}}{1-t^{2}}+\frac{1}{2} \frac{(1-t)^{6}}{1+t^{2}}+2^{6} \frac{t^{2}+t^{4}}{1-t^{4}}\right) \\
& -\frac{t^{4}\left(1+t^{2}\right)}{1-t^{2}}\left((1+t)^{6}+2^{6}\left(t^{2}+t^{4}\right)\right) .
\end{aligned}
$$

See [Ki1 for the Betti number computation of the partial desingularization of $\mathcal{M}$, the moduli space without fixing determinant.

Furthermore, we can refine the above computation to get the Hodge-Deligne polynomial for $\mathcal{N}_{2}$, since the observation in $\S 14$ of [Ki4] tells us that the morphisms involved in the above Betti number computation are strictly compatible with the mixed Hodge structures. By the gauge-theoretic computation of [AB], the HodgeDeligne series for the equivariant cohomology $H_{G}^{*}\left(\mathfrak{R}^{s s}\right)$ is 3

$$
\frac{\left(1-u^{2} v\right)^{3}\left(1-u v^{2}\right)^{3}-(u v)^{4}(1-u)^{3}(1-v)^{3}}{(1-u v)\left(1-(u v)^{2}\right)} .
$$

Blowing up along $G Z_{S L(2)}^{s s}$ and deleting the unstable part amounts to adding

$$
2^{6}\left(\frac{u v+(u v)^{2}+\cdots+(u v)^{8}}{1-(u v)^{2}}-\frac{(u v)^{5}\left(1+u v+(u v)^{2}\right)}{1-u v}\right),
$$

and blowing up along $G \tilde{Z}_{\mathbb{C}^{*}}^{s s}$ and deleting unstable points amounts to adding

$$
\begin{aligned}
& \left(u v+(u v)^{2}+(u v)^{3}\right)\left(\frac{1}{2} \frac{(1-u)^{3}(1-v)^{3}}{1-u v}+\frac{1}{2} \frac{(1+u)^{3}(1+v)^{3}}{1+u v}+2^{6} \frac{u v+(u v)^{2}}{1-(u v)^{2}}\right) \\
& -\frac{(u v)^{2}(1+u v)}{1-u v}\left((1-u)^{3}(1-v)^{3}+2^{6}\left(u v+(u v)^{2}\right)\right) .
\end{aligned}
$$

\footnotetext{
${ }^{3}$ The recent article [EK] by Earl and Kirwan contains detailed arguments for the Hodge number computation of the equivariant cohomology.
} 
Therefore, we get

$$
\begin{aligned}
& E\left(\mathcal{N}_{2}\right)=\frac{\left(1-u^{2} v\right)^{3}\left(1-u v^{2}\right)^{3}-(u v)^{4}(1-u)^{3}(1-v)^{3}}{(1-u v)\left(1-(u v)^{2}\right)} \\
& +2^{6}\left(\frac{u v+(u v)^{2}+\cdots+(u v)^{8}}{1-(u v)^{2}}-\frac{(u v)^{5}\left(1+u v+(u v)^{2}\right)}{1-u v}\right) \\
& +\left(u v+(u v)^{2}+(u v)^{3}\right)\left(\frac{1}{2} \frac{(1-u)^{3}(1-v)^{3}}{1-u v}+\frac{1}{2} \frac{(1+u)^{3}(1+v)^{3}}{1+u v}+2^{6} \frac{u v+(u v)^{2}}{1-(u v)^{2}}\right) \\
& \quad-\frac{(u v)^{2}(1+u v)}{1-u v}\left((1-u)^{3}(1-v)^{3}+2^{6}\left(u v+(u v)^{2}\right)\right) .
\end{aligned}
$$

Notice that (15) reduces to (14) if we put $u=v=-t$.

In this context, $D_{1}^{\prime}$ is the disjoint union of $2^{6}$ copies of $\mathbb{P}\left(s l(2)^{3}\right) / / S L(2)$ and $\tilde{D}_{1}^{\prime}$ is its partial desingularization. The algorithm in [Ki3] gives us

$$
E\left(\tilde{D}_{1}^{\prime}\right)=2^{6}\left(1+u v+(u v)^{2}\right)\left(1+u v+(u v)^{2}+(u v)^{3}\right) .
$$

The normal bundle to $G \tilde{Z}_{\mathbb{C}^{*}}^{s s}$ has rank $2 g-2=4$, as we saw in (4). As $G \tilde{Z}_{\mathbb{C}^{*}}^{s s} \cong$ $G \times_{N^{\mathbb{C}^{*}}} \tilde{Z}_{\mathbb{C}^{*}}^{s s}$ from [Ki3] and the normal bundle can be written similarly, the quotient of the normal bundle by $G$ is the quotient of its restriction to $\tilde{Z}_{\mathbb{C}^{*}}^{s s}$ by the action of $N^{\mathbb{C}^{*}}$. If we first take the quotient by the identity component $N_{0}^{\mathbb{C}^{*}}$ of $N^{\mathbb{C}^{*}}$, we get a $\mathbb{C}^{4} / / \mathbb{C}^{*}$-bundle over $\widetilde{J a c}$, the blow-up of Jac along $\mathbb{Z}_{2}^{6}$, since $\widetilde{Z}_{\mathbb{C}^{*}} / / N_{0}^{\mathbb{C}^{*}} \cong \widetilde{J a c}$. Hence there is a neighborhood of $\tilde{\mathcal{K}}$ in $\mathcal{N}_{1}$, which is isomorphic to the $\mathbb{Z}_{2}$-quotient of the $\mathbb{C}^{4} / / \mathbb{C}^{*}$-bundle over $\tilde{J a c}$ because $\pi_{0}\left(N^{\mathbb{C}^{*}}\right)=\mathbb{Z}_{2}$. As we mentioned at the end of $\S 2$, the normal cone $\mathbb{C}^{4} / / \mathbb{C}^{*}$ is obtained by collapsing the zero section of the line bundle $\mathcal{O}_{\mathbb{P}^{1} \times \mathbb{P}^{1}}(-1,-1)$, and thus the exceptional divisor $D_{2}^{\prime}$ is the $\mathbb{Z}_{2}$ quotient of the $\mathbb{P}^{1} \times \mathbb{P}^{1}$ bundle over $\widetilde{J a c}$. Hence, the E-polynomial of $D_{2}^{\prime}$ is

$$
\begin{aligned}
E\left(D_{2}^{\prime}\right)= & {\left[\left((1-u)^{3}(1-v)^{3}+2^{6}\left(u v+(u v)^{2}\right)\right)(1+u v)^{2}\right]^{\mathbb{Z}_{2}} } \\
= & \left(\frac{1}{2}(1-u)^{3}(1-v)^{3}+\frac{1}{2}(1+u)^{3}(1+v)^{3}+2^{6}\left(u v+(u v)^{2}\right)\right) \\
& \times\left(1+u v+(u v)^{2}\right) \\
+ & \left(\frac{1}{2}(1-u)^{3}(1-v)^{3}-\frac{1}{2}(1+u)^{3}(1+v)^{3}\right)(u v),
\end{aligned}
$$

where $[\cdot]^{\mathbb{Z}_{2}}$ denotes the $\mathbb{Z}_{2}$-invariant part. The intersection of the two divisors $D_{2}^{\prime}$ and $\tilde{D}_{1}^{\prime}$ has $2^{6}$ components, each of which is isomorphic to a bundle over $\mathbb{P}^{2}$ with fiber $\mathbb{P}^{2}=\mathbb{P}^{1} \times_{\mathbb{Z}_{2}} \mathbb{P}^{1}$.

Now, we can compute the E-function of the smooth part $\mathcal{N}^{s}=\mathcal{N}-\mathcal{K}=\mathcal{N}_{2}-$ $D_{2}^{\prime}-\tilde{D}_{1}^{\prime}$. The E-polynomials of $\mathcal{N}_{2}$ and $\tilde{D}_{1}^{\prime}$ are (15) and (16), respectively. The E-polynomial of $D_{2}^{\prime}-\tilde{D}_{1}^{\prime}$ is

$$
\begin{aligned}
& \left(\frac{1}{2}(1-u)^{3}(1-v)^{3}+\frac{1}{2}(1+u)^{3}(1+v)^{3}-2^{6}\right)\left(1+u v+(u v)^{2}\right) \\
& +\left(\frac{1}{2}(1-u)^{3}(1-v)^{3}-\frac{1}{2}(1+u)^{3}(1+v)^{3}\right)(u v),
\end{aligned}
$$


by subtracting $E\left(D_{2}^{\prime} \cap \tilde{D}_{1}^{\prime}\right)=2^{6}\left(1+u v+(u v)^{2}\right)^{2}$ from (17). Therefore, the Epolynomial of $\mathcal{N}^{s}$ is

$$
\begin{aligned}
E\left(\mathcal{N}^{s}\right) & =E\left(\mathcal{N}_{2}\right)-E\left(\tilde{D}_{1}^{\prime}\right)-E\left(D_{2}^{\prime}-\tilde{D}_{1}^{\prime}\right) \\
& =\frac{\left(1-u^{2} v\right)^{3}\left(1-u v^{2}\right)^{3}-(u v)^{4}(1-u)^{3}(1-v)^{3}}{(1-u v)\left(1-(u v)^{2}\right)} \\
& -\frac{1}{2}\left(\frac{(1-u)^{3}(1-v)^{3}}{1-u v}+\frac{(1+u)^{3}(1+v)^{3}}{1+u v}\right) .
\end{aligned}
$$

To end this section, we consider the singular locus of $\mathcal{N}_{2}$. At a point in $D_{2}^{\prime} \backslash \tilde{D}_{1}^{\prime}$, $\mathcal{N}_{2}$ looks like a line bundle over $\mathbb{P}^{1} \times \mathbb{P}^{1}$ times $\mathbb{C}^{3}$, and hence is smooth. The singular locus thus lies in $\tilde{D}_{1}^{\prime}$, and so we restrict our concern to $X_{1}$, the blow-up of $X=s l(2)^{3} / / S L(2)$. We know from the previous section that $X_{1}$ is smooth at points in $D_{1}^{\prime} \backslash \Delta_{X}$. Hence, the singular locus of $\mathcal{N}_{2}$ lies over $\Delta$. We claim that the proper transform $\tilde{\Delta}$ of $\Delta$ is precisely the singular locus in $\mathcal{N}_{2}$. To verify our claim, we return to the local chart description.

In terms of the local chart (5), $X_{1}$ is given by the equation (6) and $\tilde{\mathcal{K}}_{X}$ is given by (8). We introduce new coordinates

$$
\begin{aligned}
& w_{1}=y_{1}, w_{2}=y_{2}-y_{4}^{2}, w_{3}=y_{3}-y_{5}^{2}, \\
& w_{4}=y_{4}, w_{5}=y_{5}, w_{6}=y_{6}-y_{4} y_{5}, w_{7}=y_{7} .
\end{aligned}
$$

Then the equation of $X_{1}$ is $w_{1}\left(w_{2} w_{3}-w_{6}^{2}\right)-w_{7}^{2}$ and $\tilde{\mathcal{K}}_{X}$ is given by $w_{2}=w_{3}=$ $w_{6}=w_{7}=0$. The blow-up along $\tilde{\mathcal{K}}_{X}$ can now be described locally as

$$
\left(t_{1}, \cdots, t_{7}\right) \rightarrow\left(t_{1}, t_{2}, t_{2} t_{3}, t_{4}, t_{5}, t_{2} t_{6}, t_{2} t_{7}\right) .
$$

Since $w_{1}\left(w_{2} w_{3}-w_{6}^{2}\right)-w_{7}^{2}=t_{2}^{2}\left(t_{1}\left(t_{3}-t_{6}^{2}\right)-t_{7}^{2}\right)$ in this chart, $X_{2}$ is given by the equation

$$
g_{12}\left(t_{1}, \cdots, t_{7}\right)=t_{1}\left(t_{3}-t_{6}^{2}\right)-t_{7}^{2} .
$$

The singular locus is, from $\nabla g_{12}=0$,

$$
t_{1}=0, t_{7}=0, t_{3}-t_{6}^{2}=0,
$$

which is the proper transform of $\Delta_{X}$ in view of the fact that $\Delta_{X}$ is $w_{1}=w_{7}=$ $0, w_{2} w_{3}-w_{6}^{2}=0$ from (77). Similarly, one can use other charts for the second blow-up to check that the proper transform $\tilde{\Delta}_{X}$ of $\Delta_{X}$ is the singular locus over the local chart (5).

In the local chart (9), $X_{1}$ is given by (10) and $\tilde{\mathcal{K}}_{X}$ by (12), while $\Delta_{X}$ is given by (11). Since we are interested in a neighborhood of $\Delta_{X} \cap \tilde{\mathcal{K}}_{X}$ where $y_{1} \neq 0$, we may assume that $y_{1} \neq 0$. We introduce new coordinates

$$
\begin{aligned}
& w_{1}=y_{1}, \quad w_{2}=y_{2}-y_{4}^{2} / y_{1}, w_{3}=y_{3}-1 / y_{1}, w_{4}=y_{4}, \\
& w_{5}=y_{5}, \quad w_{6}=y_{6}-y_{4} / y_{1}, \quad w_{7}=y_{7} .
\end{aligned}
$$

In terms of $w$-coordinates, $X_{1}$ is just $w_{1} w_{5}\left(w_{2} w_{3}-w_{6}^{2}\right)-w_{7}^{2}$ and $\tilde{\mathcal{K}}_{X}$ is $w_{2}=w_{3}=$ $w_{6}=w_{7}=0$. The blow-up map along $\tilde{\mathcal{K}}_{X}$ can be written locally as (19), for instance. One can check again that the singular locus of $\mathcal{N}_{2}$ over the local chart (99) is precisely $\tilde{\Delta}_{X}$.

By a similar computation for each local chart for $X_{1}$, we deduce that the singular locus of $\mathcal{N}_{2}$ is $\tilde{\Delta}$, as claimed. Observe from the above that $\tilde{\Delta}$ and $\tilde{D}_{1}^{\prime}$ are smooth. 


\section{THIRD BLOW-UP}

To obtain a desingularization $\tilde{\mathcal{N}}$ of $\mathcal{N}$, we blow up $\mathcal{N}_{2}$ along $\tilde{\Delta}$. Let $D_{3}$ be the exceptional divisor of this third blow-up, and let $D_{1}, D_{2}$ denote the proper transforms of $\tilde{D}_{1}^{\prime}, D_{2}^{\prime}$ respectively.

In terms of the $t$-coordinates (19) of $\mathcal{N}_{2}$, one can readily deduce from (20) and (21) that the singularity along $\tilde{\Delta}$ is just the $\left(x y=z^{2}\right)$-singularity in $\mathbb{C}^{3}$, and by blowing up along $\tilde{\Delta}$ we get a smooth variety. As one can check, the same is true for each local chart of $\mathcal{N}_{2}$. Hence, $\tilde{\mathcal{N}}$ is smooth.

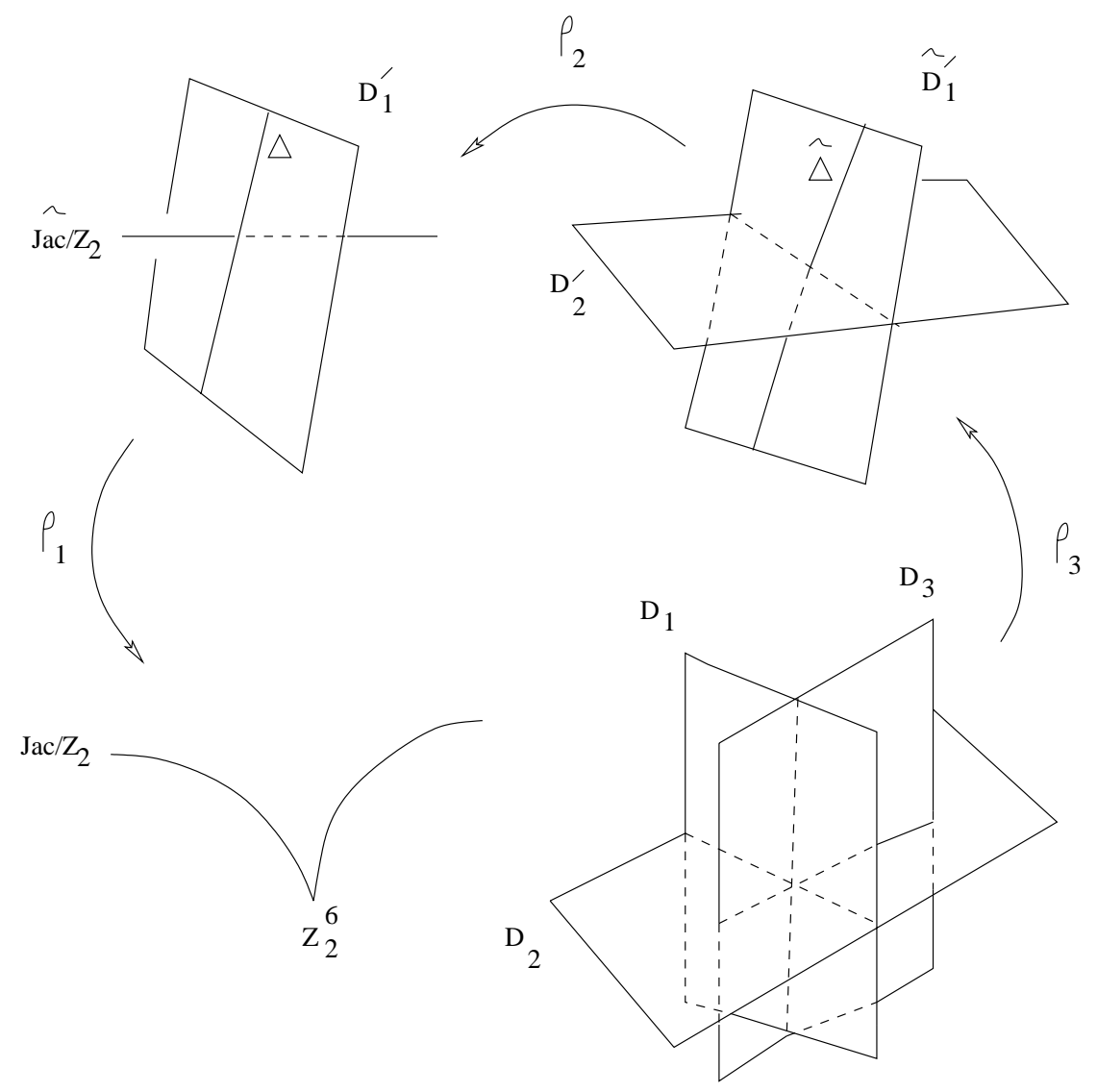

FIGURE 1.

One can also explicitly check in terms of local coordinates that the divisors $D_{1}, D_{2}, D_{3}$ are smooth divisors with only normal crossings. For instance, consider the $t$-coordinates (19) for $\mathcal{N}_{2}$ again. Before blowing up, we introduce new coordinates $r_{1}=t_{1}, r_{2}=t_{2}, r_{3}=t_{3}-t_{6}^{2}, r_{4}=t_{4}, r_{5}=t_{5}, r_{6}=t_{6}, r_{7}=t_{7}$. Then $\mathcal{N}_{2}$ is given by $r_{1} r_{3}-r_{7}^{2}=0$ and the blow-up center is $r_{1}=r_{3}=r_{7}=0$. If we consider the local description of the third blow-up, for instance,

$$
\left(\alpha_{1}, \cdots, \alpha_{6}, \alpha_{7}\right) \rightarrow\left(\alpha_{3} \alpha_{1}, \alpha_{2}, \alpha_{3}, \alpha_{4}, \alpha_{5}, \alpha_{6}, \alpha_{3} \alpha_{7}\right)
$$


then $\tilde{\mathcal{N}}$ is $\alpha_{1}-\alpha_{7}^{2}=0, D_{1}$ is $\alpha_{1}=\alpha_{7}=0, D_{2}$ is $\alpha_{2}=0=\alpha_{1}-\alpha_{7}^{2}$ and $D_{3}$ is $\alpha_{3}=0=\alpha_{1}-\alpha_{7}^{2}$. By repeating a similar computation for each chart, we see that the divisors have only normal crossings.

The desingularization process we described can be schematically summarized in Figure 1.

\section{CANONICAL Divisors}

The purpose of this section is to prove the following.

Proposition 6.1. If $\rho: \tilde{\mathcal{N}} \rightarrow \mathcal{N}$ is the desingularization described above, then $K_{\tilde{\mathcal{N}}}=\rho^{*} K_{\mathcal{N}}+4 D_{1}+D_{2}+4 D_{3}$.

We consider a differential

$$
s=\frac{d x_{1} \wedge d x_{2} \wedge d x_{3} \wedge d x_{5} \wedge d x_{6} \wedge d x_{7}}{\partial f / \partial x_{4}}
$$

on $X=s l(2)^{3} / / S L(2)$. On the smooth part of $X, s$ is not vanishing and thus the divisor of $s$ is zero. (See (1.7) in [Rei].) In terms of local coordinates, the first blow-up map $\rho_{1}$ is given by $\left(y_{1}, \cdots, y_{6}, y_{7}\right) \rightarrow\left(y_{1}, y_{1} y_{2}, \cdots, y_{1} y_{6}, y_{1} y_{7}\right)$, and we have a rational differential on $X_{1}$,

$$
s=y_{1}^{4} \frac{d y_{1} \wedge d y_{2} \wedge d y_{3} \wedge d y_{5} \wedge d y_{6} \wedge d y_{7}}{\partial g_{1} / \partial y_{4}},
$$

where $f\left(x_{1}, \cdots, x_{7}\right)=y_{1}^{2} g_{1}\left(y_{1}, \cdots, y_{7}\right)$. Hence, $K_{\mathcal{N}_{1}}=\rho_{1}^{*} K_{\mathcal{N}}+4 D_{1}^{\prime}$.

Now we switch to the $w$-coordinates $w_{1}=y_{1}, w_{2}=y_{2}-y_{4}^{2}, w_{3}=y_{3}-y_{5}^{2}, w_{4}=$ $y_{4}, w_{5}=y_{5}, w_{6}=y_{6}-y_{4} y_{5}$. Then $g_{1}=w_{1}\left(w_{2} w_{3}-w_{6}^{2}\right)-w_{7}^{2}$. The second blow-up, in terms of local coordinates, is $\left(t_{1}, \cdots, t_{7}\right) \rightarrow\left(t_{1}, t_{2}, t_{2} t_{3}, t_{4}, t_{5}, t_{2} t_{6}, t_{2} t_{7}\right)$, and we get a rational differential on $X_{2}$ :

$$
\begin{aligned}
s & =w_{1}^{4} \frac{d w_{1} \wedge d w_{2} \wedge d w_{3} \wedge d w_{5} \wedge d w_{6} \wedge d w_{7}}{\partial g_{1} / \partial w_{3}} \\
& =t_{1}^{4} t_{2} \frac{d t_{1} \wedge d t_{2} \wedge d t_{3} \wedge d t_{5} \wedge d t_{6} \wedge d t_{7}}{\partial g_{12} / \partial t_{3}},
\end{aligned}
$$

where $g_{1}\left(y_{1}, \cdots, y_{7}\right)=t_{2}^{2} g_{12}\left(t_{1}, \cdots, t_{7}\right)$. Hence,

$$
K_{\mathcal{N}_{2}}=\rho_{2}^{*} \rho_{1}^{*} K_{\mathcal{N}}+4 \tilde{D}_{1}^{\prime}+D_{2}^{\prime} .
$$

We next use the $r$-coordinates $r_{1}=t_{1}, r_{2}=t_{2}, r_{3}=t_{3}-t_{6}^{2}, r_{4}=t_{4}, r_{5}=$ $t_{5}, r_{6}=t_{6}, r_{7}=t_{7}$. Then $g_{12}=r_{1} r_{3}-r_{7}^{2}$. Finally, we blow up along $r_{1}=$ $r_{3}=r_{7}=0$. In terms of local coordinates, the blow-up is $\left(\alpha_{1}, \cdots, \alpha_{7}\right) \rightarrow$ $\left(\alpha_{3} \alpha_{1}, \alpha_{2}, \alpha_{3}, \alpha_{4}, \alpha_{5}, \alpha_{6}, \alpha_{3} \alpha_{7}\right)$ and $g_{12}=\alpha_{3}^{2}\left(\alpha_{1}-\alpha_{7}^{2}\right)$. The equation for $\tilde{\mathcal{N}}$ in the $\alpha$-coordinates is thus $g_{123}=\alpha_{1}-\alpha_{7}^{2}$, and we have a rational differential on $\tilde{X}$ :

$$
s=\alpha_{1}^{4} \alpha_{2} \alpha_{3}^{4} \frac{d \alpha_{2} \wedge d \alpha_{3} \wedge \cdots \wedge d \alpha_{7}}{\partial g_{123} / \partial \alpha_{1}} .
$$

By a similar computation for each chart, we deduce that

$$
K_{\tilde{\mathcal{N}}}=\rho^{*} K_{\mathcal{N}}+4 D_{1}+D_{2}+4 D_{3}
$$




\section{The STRINGY E-FunCTION}

We can now compute the stringy E-function of the moduli space $\mathcal{N}$.

The E-function of the smooth part is from $\S 4$ :

$$
\begin{aligned}
E\left(\mathcal{N}^{s}\right) & =E\left(\mathcal{N}_{2}\right)-E\left(\tilde{D}_{1}^{\prime}\right)-E\left(D_{2}^{\prime}-\tilde{D}_{1}^{\prime}\right) \\
& =\frac{\left(1-u^{2} v\right)^{3}\left(1-u v^{2}\right)^{3}-(u v)^{4}(1-u)^{3}(1-v)^{3}}{(1-u v)\left(1-(u v)^{2}\right)} \\
& -\frac{1}{2}\left(\frac{(1-u)^{3}(1-v)^{3}}{1-u v}+\frac{(1+u)^{3}(1+v)^{3}}{1+u v}\right) .
\end{aligned}
$$

Next, $D_{1}^{0}=D_{1}-\left(D_{2} \cup D_{3}\right)$ is $\tilde{D}_{1}^{\prime}-\tilde{\Delta}-\tilde{D}_{1}^{\prime} \cap D_{2}^{\prime}$. Since each component of $\tilde{D}_{1}^{\prime} \cap D_{2}^{\prime}$ is a $\mathbb{P}^{2}$-bundle over $\mathbb{P}^{2}$ and each component of $\tilde{\Delta}-D_{2}^{\prime}$ is $\mathbb{P}^{2} \times_{\mathbb{Z}_{2}} \mathbb{P}^{2}-\mathbb{P}^{2}$, $E\left(D_{1}^{0}\right)$ is (16) minus $E\left(\mathbb{P}^{2} \times \mathbb{P}^{2}\right)$ and $2^{6}\left[\left(1+u v+(u v)^{2}\right)^{2}\right]^{\mathbb{Z}_{2}}-2^{6}\left(1+u v+(u v)^{2}\right)$. Hence,

$$
E\left(D_{1}^{0}\right) \frac{u v-1}{(u v)^{5}-1}=2^{6}\left((u v)^{5}-(u v)^{2}\right) \frac{u v-1}{(u v)^{5}-1} .
$$

Since $D_{2}^{0}=D_{2}-\left(D_{1} \cup D_{3}\right)=D_{2}^{\prime}-\tilde{D}_{1}^{\prime}$, the E-function of $D_{2}^{0}$ is (17) minus the E-function of $D_{2}^{\prime} \cap \tilde{D}_{1}^{\prime}, 2^{6}\left(1+u v+(u v)^{2}\right)^{2}$. Hence,

$$
\begin{aligned}
& E\left(D_{2}^{0}\right) \frac{u v-1}{(u v)^{2}-1} \\
& =\left(\frac{1}{2}(1-u)^{3}(1-v)^{3}+\frac{1}{2}(1+u)^{3}(1+v)^{3}-2^{6}\right)\left(1+u v+(u v)^{2}\right) \frac{u v-1}{(u v)^{2}-1} \\
& +\left(\frac{1}{2}(1-u)^{3}(1-v)^{3}-\frac{1}{2}\left(1+u^{3}(1+v)^{3}\right)(u v) \frac{u v-1}{(u v)^{2}-1} .\right.
\end{aligned}
$$

As $D_{3} \cap D_{1}$ is isomorphic to $\tilde{\Delta}$ and a component of $D_{3} \cap D_{2}$ is a $\mathbb{P}^{1}$-bundle over $\tilde{\Delta} \cap D_{2}^{\prime}$, we see that the E-function of $D_{3}^{0}$ is $2^{6}$ times the E-function of a $\mathbb{P}^{1}$-bundle over $\tilde{\Delta}$ minus $E(\tilde{\Delta})$ and $2^{6}$ times $E\left(\left(\mathbb{P}^{1}-p t\right) \times \mathbb{P}^{1} \times \mathbb{P}^{2}\right)$. Hence,

$$
E\left(D_{3}^{0}\right) \frac{u v-1}{(u v)^{5}-1}=2^{6}\left((u v)^{3}+(u v)^{4}+(u v)^{5}\right) \frac{u v-1}{(u v)^{5}-1} .
$$

Notice that $D_{12}^{0}=D_{1} \cap D_{2}-D_{3}=\tilde{D}_{1}^{\prime} \cap D_{2}^{\prime}-\tilde{\Delta}$ is the disjoint union of $2^{6}$ copies of a $\left(\mathbb{P}^{2}-\mathbb{P}^{1}\right)$-bundle over $\mathbb{P}^{2}$. Hence,

$$
E\left(D_{12}^{0}\right) \frac{u v-1}{(u v)^{5}-1} \frac{u v-1}{(u v)^{2}-1}=2^{6}\left((u v)^{2}+(u v)^{3}+(u v)^{4}\right) \frac{u v-1}{(u v)^{5}-1} \frac{u v-1}{(u v)^{2}-1} .
$$

Also, $D_{13}^{0}=D_{1} \cap D_{3}-D_{2}$ is $\tilde{\Delta}$ minus $2^{6} \mathbb{P}^{1}$-bundles over $\mathbb{P}^{2}$. Hence,

$$
E\left(D_{13}^{0}\right)\left(\frac{u v-1}{(u v)^{5}-1}\right)^{2}=2^{6}\left((u v)^{2}+(u v)^{3}+(u v)^{4}\right)\left(\frac{u v-1}{(u v)^{5}-1}\right)^{2} .
$$

Finally, a component of $D_{23}^{0}=D_{2} \cap D_{3}-D_{1}$ is a $\left(\mathbb{P}^{1}-p t\right)$-bundle over a $\mathbb{P}^{1}$. bundle over $\mathbb{P}^{2}$, and a component of $D_{123}^{0}=D_{1} \cap D_{2} \cap D_{3}$ is a $\mathbb{P}^{1}$-bundle over $\mathbb{P}^{2}$. Therefore,

$$
E\left(D_{23}^{0}\right) \frac{u v-1}{(u v)^{5}-1} \frac{u v-1}{(u v)^{2}-1}=2^{6}\left(u v+(u v)^{2}+(u v)^{3}\right) \frac{u v-1}{(u v)^{5}-1}
$$

and

$$
E\left(D_{123}^{0}\right)\left(\frac{u v-1}{(u v)^{5}-1}\right)^{2} \frac{u v-1}{(u v)^{2}-1}=2^{6}\left(1+u v+(u v)^{2}\right)\left(\frac{u v-1}{(u v)^{5}-1}\right)^{2} .
$$


Putting together all the pieces above, we get from formula (11) that

$$
\begin{aligned}
E_{s t}(\mathcal{N})= & \frac{\left(1-u^{2} v\right)^{3}\left(1-u v^{2}\right)^{3}-(u v)^{4}(1-u)^{3}(1-v)^{3}}{(1-u v)\left(1-(u v)^{2}\right)} \\
& -\frac{(u v)^{2}}{2}\left(\frac{(1-u)^{3}(1-v)^{3}}{1-u v}-\frac{(1+u)^{3}(1+v)^{3}}{1+u v}\right) \\
& +2^{6}(u v)^{5}\left(1+u v+(u v)^{2}\right)\left(1+(u v)^{2}\right)\left(\frac{u v-1}{(u v)^{5}-1}\right)^{2} .
\end{aligned}
$$

This satisfies the Poincaré duality (2), which serves as a check for our result. Notice that it is not a polynomial.

To prove Corollary 1.2. let $D_{j, X}$ be the divisors in $\tilde{X}$ corresponding to $D_{j}$. Then, from the above, we have

$$
\begin{gathered}
E\left(D_{1, X}^{0}\right) \frac{u v-1}{(u v)^{5}-1}=\left((u v)^{5}-(u v)^{2}\right) \frac{u v-1}{(u v)^{5}-1}, \\
E\left(D_{2, X}^{0}\right) \frac{u v-1}{(u v)^{2}-1}=\left((u v)^{3}-1\right)\left(1+u v+(u v)^{2}\right) \frac{u v-1}{(u v)^{2}-1}, \\
E\left(D_{3, X}^{0}\right) \frac{u v-1}{(u v)^{5}-1}=\left((u v)^{3}+(u v)^{4}+(u v)^{5}\right) \frac{u v-1}{(u v)^{5}-1}, \\
E\left(D_{12, X}^{0}\right) \frac{u v-1}{(u v)^{5}-1} \frac{u v-1}{(u v)^{2}-1}=\left((u v)^{2}+(u v)^{3}+(u v)^{4}\right) \frac{u v-1}{(u v)^{5}-1} \frac{u v-1}{(u v)^{2}-1}, \\
E\left(D_{13, X}^{0}\right)\left(\frac{u v-1}{(u v)^{5}-1}\right)^{2}=\left((u v)^{2}+(u v)^{3}+(u v)^{4}\right)\left(\frac{u v-1}{(u v)^{5}-1}\right)^{2}, \\
E\left(D_{23, X}^{0}\right) \frac{u v-1}{(u v)^{5}-1} \frac{u v-1}{(u v)^{2}-1}=\left(u v+(u v)^{2}+(u v)^{3}\right) \frac{u v-1}{(u v)^{5}-1},
\end{gathered}
$$

and

$$
E\left(D_{123, X}^{0}\right)\left(\frac{u v-1}{(u v)^{5}-1}\right)^{2} \frac{u v-1}{(u v)^{2}-1}=\left(1+u v+(u v)^{2}\right)\left(\frac{u v-1}{(u v)^{5}-1}\right)^{2} .
$$

By putting them together, we get

$$
\begin{aligned}
E_{s t}\left(\mathbb{C}^{9} / / S L(2)\right) & =E\left(\left[\mathbb{C}^{9} / / S L(2)\right]^{s}\right)+\frac{(u v)^{3}\left(1+u v+(u v)^{2}\right)}{1+u v} \\
& +(u v)^{5}\left(1+u v+(u v)^{2}\right)\left(1+(u v)^{2}\right)\left(\frac{u v-1}{(u v)^{5}-1}\right)^{2},
\end{aligned}
$$

where $\left[\mathbb{C}^{9} / / S L(2)\right]^{s}$ denotes the smooth part of $\mathbb{C}^{9} / / S L(2)$.

Remark 7.1. If we denote by $\mathcal{M}$ the moduli space of rank 2 semistable bundles of even degree over a Riemann surface of genus 3 (without fixing the determinant), the stringy E-function is

$$
\begin{aligned}
E_{s t}(\mathcal{M}) & =(1-u)^{3}(1-v)^{3}\left\{\frac{\left(1-u^{2} v\right)^{3}\left(1-u v^{2}\right)^{3}-(u v)^{4}(1-u)^{3}(1-v)^{3}}{(1-u v)\left(1-(u v)^{2}\right)}\right. \\
& -\frac{(u v)^{2}}{2}\left(\frac{(1-u)^{3}(1-v)^{3}}{1-u v}-\frac{(1+u)^{3}(1+v)^{3}}{1+u v}\right) \\
& \left.+(u v)^{5}\left(1+u v+(u v)^{2}\right)\left(1+(u v)^{2}\right)\left(\frac{u v-1}{(u v)^{5}-1}\right)^{2}\right\} .
\end{aligned}
$$

We just sketch the computation, and leave the details to the reader. The determinant map det $: \mathcal{M} \rightarrow J a c$ is a fibration with fiber $\mathcal{N}$, and $\mathcal{M}$ has the same 
singularities as $\mathcal{N}$. So we need three blow-ups, exactly as in $\S \S 3,4,5$, and the discrepancy divisor is given as in Proposition 6.1. It is now easy to modify the computation to get

$$
\begin{gathered}
E\left(\mathcal{M}^{s}\right)=(1-u)^{3}(1-v)^{3}\left[\frac{\left(1-u^{2} v\right)^{3}\left(1-u v^{2}\right)^{3}-(u v)^{4}(1-u)^{3}(1-v)^{3}}{(1-u v)\left(1-(u v)^{2}\right)}\right. \\
\left.-\frac{1}{2}\left(\frac{(1-u)^{3}\left(1-v^{3}\right.}{1-u v}+\frac{(1+u)^{3}(1+v)^{3}}{1+u v}\right)\right] \\
E\left(D_{1, \mathcal{M}}^{0}\right) \frac{u v-1}{(u v)^{5}-1}=(1-u)^{3}(1-v)^{3}\left((u v)^{5}-(u v)^{2}\right) \frac{u v-1}{(u v)^{5}-1} \\
E\left(D_{2, \mathcal{M}}^{0}\right) \frac{u v-1}{(u v)^{2}-1} \\
=(1-u)^{3}(1-v)^{3} \\
\times\left[\left(\frac{1}{2}(1-u)^{3}(1-v)^{3}+\frac{1}{2}(1+u)^{3}(1+v)^{3}-1\right)\left(1+u v+(u v)^{2}\right)\right. \\
\left.+\left(\frac{1}{2}(1-u)^{3}(1-v)^{3}-\frac{1}{2}(1+u)^{3}(1+v)^{3}\right)(u v)\right] \frac{u v-1}{(u v)^{2}-1}, \\
E\left(D_{3, \mathcal{M}}^{0}\right) \frac{u v-1}{(u v)^{5}-1}=(1-u)^{3}(1-v)^{3}\left((u v)^{3}+(u v)^{4}+(u v)^{5}\right) \frac{u v-1}{(u v)^{5}-1} \\
E\left(D_{12, \mathcal{M})}^{0} \frac{u v-1}{(u v)^{5}-1} \frac{u v-1}{(u v)^{2}-1}\right. \\
=(1-u)^{3}(1-v)^{3}\left((u v)^{2}+(u v)^{3}+(u v)^{4}\right) \frac{u v-1}{(u v)^{5}-1} \frac{u v-1}{(u v)^{2}-1}, \\
E\left(D_{23, \mathcal{M})}^{0} \frac{u v-1}{(u v)^{5}-1} \frac{u v-1}{(u v)^{2}-1}=(1-u)^{3}(1-v)^{3}\left(u v+(u v)^{2}+(u v)^{3}\right) \frac{u v-1}{(u v)^{5}-1}\right. \\
E\left(D_{13, \mathcal{M}}^{0}\right)\left(\frac{u v-1}{(u v)^{5}-1}\right)^{2}=(1-u)^{3}(1-v)^{3}\left((u v)^{2}+(u v)^{3}+(u v)^{4}\right)\left(\frac{u v-1}{(u v)^{5}-1}\right)^{2} \\
\end{gathered}
$$

and

$$
\begin{aligned}
& E\left(D_{123, \mathcal{M}}^{0}\right)\left(\frac{u v-1}{(u v)^{5}-1}\right)^{2} \frac{u v-1}{(u v)^{2}-1} \\
& \quad=(1-u)^{3}(1-v)^{3}\left(1+u v+(u v)^{2}\right)\left(\frac{u v-1}{(u v)^{5}-1}\right)^{2} .
\end{aligned}
$$

Combining these, we get (22).

\section{ACKNOWLEDGEMENTS}

I would like to thank Tamas Hausel, Michael Thaddeus, Frances Kirwan and Jun Li for useful discussions. Also, I am grateful to the referee for comments and suggestions which led to improvement in the exposition. 


\section{REFERENCES}

[AB] M. Atiyah and R. Bott. Yang-Mills equations over Riemann surfaces. Philos. Trans. Royal Soc. London A 308, 1982. Pages 523-615. MR 85k:14006

[Bat] V. Batyrev. Stringy Hodge numbers of varieties with Gorenstein canonical singularities. In Integrable systems and algebraic geometry (Kobe/Kyoto,1997), 1998. Pages 1-32. MR 2001a:14039

[Cra] A. Craw. An introduction to motivic integration. Preprint, math.AG/9911179.

[DL1] J. Denef and F. Loeser. Germs of arcs on singular varieties and motivic integration. Invent. Math. 135, no 1, 1999. Pages 201-232. MR 99k:14002

[DL2] J. Denef and F. Loeser. Motivic Igusa zeta functions. J. Algebraic Geometry 7, 1998. Pages 505-537. MR 99j:14021

[DN] J.-M. Drezet and M. Narasimhan. Groupe de Picard des variétés de modules de fibrés semistables sur les courbes algébriques. Invent. Math. 97, 1989. Pages 53-94. MR 90d:14008

[EK] R. Earl and F. Kirwan. The Hodge numbers of the moduli spaces of vector bundles over a Riemann surface. Quart. J. Math. 51 (2000), 465-483. MR 2001m:14018

[Hue] J. Huebschmann. Poisson Geometry of flat connections for SU(2)-bundles on surfaces. Math. Zeit. 221, 1996. Pages 243-259. MR 97f:58029

[Ki1] F. Kirwan. On the homology of compactifications of moduli spaces of vector bundles over a Riemann surface. Proc. London Math. Soc. 53, 1986. Pages 237-266. MR 88e:14012

[Ki2] F. Kirwan. On spaces of maps from Riemann surfaces to Grassmannians and applications to the cohomology of moduli spaces of vector bundles. Ark. Mat. 24, 1986. Pages 221-275. MR 88h:14014

[Ki3] F. Kirwan. Partial desingularizations of quotients of nonsingular varieties and their Betti numbers. Ann. Math. 122, 1985. Pages 41-85. MR 87a:14010

[Ki4] F. Kirwan. Cohomology of quotients in algebraic and symplectic geometry. Mathematical Notes 31. Princeton, 1985. MR 86i:58050

[Loo] E. Looijenga. Motivic measures. Sém. Bourbaki 1999/2000, Astérisque, No. 276 (2002), 267-297.

[New] P. Newstead. Introduction to moduli problems and orbit spaces. Tata Institute Lecture Note, 1978. MR 81k:14002

[Rei] M. Reid. Young person's guide to canonical singularities. In Algebraic Geometry Bowdoin. Vol.46, Proc. Sympos. Pure Math., American Mathematical Society, Providence, RI, 1987, pages 345-414. MR 89b:14016

[Ses] C. Seshadri. Fibrés vectoriels sur les courbes algébriques. Astérisque 96, (1982). MR 85b:14023

[Wey] H. Weyl. The classical groups. Princeton University Press, 1946. MR 98k:01049 (reprint)

Department of Mathematics, Stanford University, Stanford, California 94305

E-mail address: kiem@math.stanford.edu

Current address: Department of Mathematics, Seoul National University, San 56-1, Seoul 151-747, Korea 